\title{
GUÍA CURRICULAR. UN SISTEMA MULTIMEDIA PARA LA FORMACIÓN DE PROFESORES EN LA EDUCACIÓN FÍSICA BÁSICA
}

\section{Iván Darío Uribe Pareja, Didier Fernando Gaviria Cortés $(*)$}

SÍNTESIS: La Guía curricular es un sistema multimedia para la formación de maestros en educación física desde el nivel preescolar hasta el grado noveno, y se constituye en una propuesta alternativa de cualificación y de transformación educativa en el área.

Se soporta por medio de un CD-ROM, y está compuesta por varias herramientas tecnológicas (chat, foro, correo electrónico, videos y módulos impresos que configuran el desarrollo de la planeación de la educación física básica).

Tiene como finalidad aprovechar las experiencias, las prácticas, los conocimientos y las motivaciones del maestro, para establecer diálogos de saberes y para elaborar, a través de medios tecnológicos, construcciones curriculares colectivas y contextualizadas histórica, social y culturalmente. 


\section{INTRODUCCIÓN}

Los sistemas de capacitación (cursos presenciales y semipresenciales, programas microcurriculares y centros de educación física con encuentros de capacitación para maestros) en el departamento de Antioquia y en Colombia, han cumplido un ciclo en el cual se han desarrollado procesos significativos de formación de maestros; sin embargo, considerando los vertiginosos cambios que se han presentado en las comunicaciones y en el desarrollo científico y tecnológico, es necesario desarrollar procesos complementarios de capacitación que puedan responder a las exigencias del conocimiento, a la educación y a la pedagogía en las relaciones universidad-empresa-Estado del momento actual, de manera que el desarrollo contribuya de verdad a la solución de los problemas de las comunidades, sobre todo en el área de la educación física.

En este sentido, se ha presentado como alternativa de formación de maestros en educación física la Guía curricular. Un sistema multimedia para la formación de profesores en la educación física básica (preescolar y grados 1 al 9$)^{1}$.

Esta Guía, en su estructura de contenidos, incluye aspectos legales, fundamentos teóricos, metodológicos y evaluativos, un glosario de términos, propuestas microcurriculares por grados, y un manual para el uso de los medios virtuales básicos para facilitar al docente la interacción con sus pares académicos, con grupos de investigación, con comunidades educativas, y con la Universidad de Antioquia como institución de educación superior interesada en dinamizar el desarrollo disciplinar y profesional en el área.

\subsection{ANTECEDENTES DE LA ESTRUCTURACIÓN DE LA GUÍA CURRICULAR}

La Guía nació en 1996 como trabajo de grado para una especialización en computación para la docencia, y su nombre inicial fue Software en pedagogía: la motricidad para la educación física escolar.

${ }^{1}$ Premio Nacional en el Séptimo Congreso Colombiano de Informática Educativa. Primer puesto en la categoría de Experiencias Docentes, 2004. Mención como mejor trabajo de proyección a la comunidad del Instituto Universitario de Educación Física de la Universidad de Antioquia, 2004. 
Fue una propuesta construida en hipertexto, usando un programa llamado Hiperhel per, y con la pretensión de brindar una herramienta para la formación de maestros de educación física.

La propuesta se constituyó en la base para participar en una convocatoria para una plaza docente en la Universidad de Antioquia en 1999, y en el 2003 se retomó el proyecto. En ese año se formó un equipo de trabajo con un comunicador y con especialistas en el área de sistemas, además de profesores universitarios con experiencia docente en educación física preescolar y básica.

Trabajaron en equipo durante un año en la construcción del proyecto, lo que implicó la constitución de subgrupos con asignación de tareas específicas relacionadas, por un lado, con asuntos técnicos, y, por otro, con la comunicación y con la pedagogía de la educación virtual y el equipo de profesores de educación física, con el cual fue necesario emprender un proceso de formación para hacer una transferencia adecuada de las propuestas y ponerlas en el escenario de la educación virtual.

\section{FUNDAMENTACIÓN TEÓRICA}

La educación física básica requiere una transformación de fondo que tenga en cuenta los avances científicos y tecnológicos de la disciplina, una transformación en la cual exista una interacción permanente con otras áreas del conocimiento, y, a la vez, con los intereses y las necesidades de las comunidades.

Para lograr la transformación manifiesta se requieren profesores contextualizados histórica y culturalmente, con acceso a formación y a información frontera para adquirir los conocimientos fundamentales de la disciplina, que faciliten la elaboración de propuestas educativas pertinentes a la realidad social y cultural con la que interactúan.

La Guía curricular, como sistema virtual para la formación del profesorado en educación física, se convirtió en una estrategia de capacitación permanente que potenciara la reflexión de la práctica educativa como un insumo para el desarrollo de procesos de investigación que generaran los conocimientos necesarios para promover una 
educación encaminada hacia el mejoramiento de los estilos, de los modos y de las condiciones de vida de la población.

El proyecto, en su estructura epistémica, está fundamentado básicamente en la corriente integracionista de la educación física (formación integral, bases teóricas de la educación física, motricidad, potenciación de las capacidades humanas, como la autonomía, la autoestima, la solidaridad, la creatividad, la felicidad y la tolerancia), y su orientación pedagógico-metodológica, evaluativa y didáctica se apoya en las pedagogías activas.

Desde esta estructura pedagógica y didáctica se propone la problematización de la educación física. El propósito general que se busca conseguir está centrado en la formación integral, y, en tal sentido, se propone el logro de competencias axiológicas (valores), cognitivas (interpretar, comprender, argumentar), investigativas (crear, innovar, construir, proponer) y operativas (saber hacer).

Los contenidos fundamentales de la Guía propuestos en el sistema están estructurados en las bases conceptuales, en la metodología, en la evaluación y en las bases legales. En la fundamentación teórica y conceptual se consideran las capacidades psicomotoras, las sociomotoras, las físicomotoras, las perceptivomotoras ylas coordinativas; las habilidades motrices, las orientaciones generales sobre educación física adaptada, la competencia motriz, y elementos básicos sobre la educabilidad y la enseñabilidad. En el campo de la metodología de la educación física se realiza un tratamiento específico de los métodos y de los estilos de enseñanza de la disciplina, expuestos en unidades didácticas, y en el área de la evaluación se consideran los conceptos de evaluación, los logros, los indicadores, las competencias y los criterios de evaluación, en tanto en bases legales se atiende a planes de desarrollo y a legislación en el área.

Finalmente, desde el componente administrativo de la docencia, se presentan orientaciones para la planeación a nivel macro y microcurricular, la construcción del proyecto integral de área de la institución educativa, y el plan de aula.

En síntesis, la Guía se estructura con la pretensión de constituirse en un soporte para que las diferentes comunidades educativas desarrollen procesos de análisis y de reflexión orientados a potenciar competencias formativas, cognitivas, sociales, tecnológicas e 
investigativas, yasí empoderarse de la construcción y del diseño curricular de la educación física en la educación básica, como un elemento indispensable que se requiere para la construcción de un sistema de educación física que responda a los intereses, a las motivaciones y a las necesidades de las diferentes comunidades.

\section{METODOLOGÍA}

La investigación participativa como propuesta metodológica que involucra a los educadores en la construcción del conocimiento y en la solución de sus problemas, para emprender las acciones de cambio y de transformación necesarias.

Se eligió esta metodología por combinar investigación, educación-aprendizaje y acción, y para involucrar a los maestros en el proyecto de investigación desde el planteamiento del problema, pasando por la interpretación de los descubrimientos, hasta la discusión de las alternativas de solución a los problemas, a las acciones modificadoras, a los proyectos en desarrollo y a la generación de nuevas preguntas como elementos para emprender otras investigaciones.

Según Rojas, «la investigación y la ciencia deben estar al servicio de la colectividad; busca ayudarle a resolver sus problemas y necesidades y ayudar a planificar su vida» $(2002$, p. 2).

En esencia, se abordaron los momentos de problematización o de investigación, de tematización y de implementación pedagógica o de acción, propuestos por Marcela Gajardo y J oão Bosco Pinto.

\subsection{MOMENTO UNO: PROBLEMATIZACIÓN}

Este momento se estudió por la configuración del conocimiento como elemento básico para la comprensión y para la significación del problema. Para ello se abordaron las siguientes fases:

- Fase de elaboración teórico-conceptual. Es una fase de indagación, reflexión, estudio y construcción del referente conceptual de la propuesta. 
- Fase de selección de actores y de escenarios del proceso. Se determinaron las características de los docentes y los escenarios de intercambio de conocimiento. Se eligieron siete profesores de la Universidad y un estudiante de educación física, y como escenarios se definieron encuentros con coordinadores de los centros de educación física.

Algunos procedimientos empleados para definir los actores y los escenarios fueron:

- Entrevistas con los docentes y con los estudiantes.

- Presentación de la idea al equipo de trabajo y a los directivos del Instituto Universitario de Educación Física de la Universidad de Antioquia.

- Acuerdos de tiempos y de compromisos con el equipo de trabajo.

- Fase de interpretación y de comprensión. Se socializó el proyecto con el equipo seleccionado, y, de forma colectiva, se reconfiguraron el programa y el cronograma de trabajo; además, se definieron los niveles educativos que había que desarrollar en la propuesta de Guía curricular.

Los instrumentos que se usaron fueron: el análisis documental, los talleres en instituciones educativas, la observación de clases y los grupos de conversación.

\subsection{MOMENTO DOS: TEMATIZACIÓN}

- Fase de análisis teórico. Se estudiaron y se analizaron las informaciones y las experiencias iniciales recolectadas con el equipo de trabajo.

- Fase de selección temática. Se seleccionaron las informaciones y las experiencias básicas para la construcción de la Guía curricular.

- Fase de construcción del proyecto de Guía curricular para la educación física básica.

- Fase de socialización: En el proceso de trabajo, y de manera permanente, se socializaron y se evaluaron, junto con los actores del equipo de trabajo y con los coordinadores de los 
centros de educación física, las construcciones transitorias, y se continuó con el desarrollo del proyecto valorando las observaciones, las evaluaciones y los nuevos aportes de los diferentes actores del proceso de construcción.

\subsection{MOMENTO TRES: DESARROLLO PEDAGÓGICO O DE CAPACITACIÓN Y FORMACIÓN DE DOCENTES DE EDUCACIÓN FISICA EN LA EDUCACIÓN BÁSICA}

El equipo de docentes de la Universidad emprendió un proceso de formación de docentes en ocho subregiones del departamento de Antioquia

- Fase de configuración de la acción educativa. Con los profesores de educación básica se establecieron procesos de comunicación, valiéndose de medios virtuales como el foro, el chat y el correo electrónico, contribuyendo así con observaciones y con anotaciones dirigidas al emprendimiento de acciones que pudieran mejorar la capacitación y la Guía curricular.

- Fase de construcción de ideas para la elaboración de proyectos Integrales deárea en educación física para las instituciones educativas, y de planes educativos municipales y subregionales con la participación de docentes y de entrenadores.

- Fase de desarrollo y evaluación: Se ha continuado con procesos de formación y con asesorías utilizando medios virtuales.

\section{RESULTADOS DE LOS PROCESOS DE FORMACIÓN}

En entrevistas y seguimientos del proceso de desarrollo de las acciones emprendidas por los maestros se encontraron los siguientes elementos:

Los maestros del área de educación física del departamento de Antioquia identificaron los medios virtuales como una estrategia y como 
un medio de formación permanente, que contribuye a su cualificación y a mejorar la calidad de la educación física en la educación básica.

Los medios tecnológicos virtuales contribuyen a mejorar la comunicación, el intercambio de conocimientos y la construcción de proyectos colectivos entre los maestros de educación física.

La Guía se ha constituido en referente teórico y en medio permanente de consulta para la construcción curricular del área de educación física por parte de los maestros que han participado en los procesos de capacitación para el manejo del sistema.

Los maestros se están apoyand o en la Guía para la estructuración de los proyectos Integrales del área de educación física, de los planes educativos municipales y de los planes subregionales en educación física y deportes.

La Guía se ha convertido en un eje transversal en los procesos educativos de algunas instituciones de educación básica.

En la experiencia aplicada de los procesos de formación de maestros se ha encontrado que, en sus interacciones, los maestros, por medio de diálogos de saberes, ponen en el escenario de la discusión las experiencias prácticas y los conocimientos en la acción.

Los medios tecnológicos virtuales integrados a encuentros colectivos como estrategias de formación y de capacitación, contribuyen y facilitan la construcción de los proyectos integrales de área y de los planes de aula en educación física.

Se requiere de dotación y de capacitación de medios tecnológicos modernos que permitan a los maestros acceder a nuevas formas de capacitación y de formación.

Es necesario emprender procesos de transformación en la cultura escolar en relación con el uso constante de los medios virtuales por los maestros.

El conocimiento y el desarrollo de habilidades en el manejo de herramientas tecnológicas por los docentes motiva el interés por el acceso a la información de frontera, y por el desarrollo de intercambio de saberes en el área. 
El sistema de capacitación a través de medios virtuales contribuye al desarrollo de motivaciones que dotan de sentido teórico-práctico, metodológico, pedagógico, organizativo y administrativo a la construcción colectiva para la elaboración de propuestas educativas en educación física, contextualizadas histórica, social y culturalmente.

\section{PRODUCTOS EN EL DESARROLLO DEL PROYECTO}

Capacitación en el manejo de la Guía y en medios virtuales de 940 maestros de educación física de las nueve subregiones del departamento de Antioquia.

Introducción a la elaboración colectiva por municipios y por subregiones de propuestas curriculares pertinentes y contextualizadas para la formación de profesores en el área de educación física.

El diseño de una Guía curricular en educación física estructurada desde las bases legales y desde los fundamentos teóricos del área, como un sistema multimedia para la capacitación de maestros en el área de educación física, que contiene: $C D$, chat, foro y correo electrónico, instalados en la página del Instituto Universitario de Educación Física de la Universidad de Antioquia, y, además, tres textos².

Tres libros en él área de educación física:

- Módulo uno: Fundamentos teóricos de la educación física.

- Módulo dos: Unidades didácticas para el preescolar y la primaria básica (grados uno a quinto).

- Módulo tres: Unidades didácticas para los grados de la secundaria básica (grados seis a nueve).

Estado del uso en Colombia en la aplicación y la utilización de las TIC en el sistema educativo:

\footnotetext{
${ }^{2}$ Los productos más sobresalientes son: el CD interactivo, que incluye vídeo, fotografía, audio, diccionario básico, foro, chat, correo electrónico, direcciones sobre educación física, y una construcción teórica sobre epistemología de la educación física; metodología, evaluación, fundamentos legales; educación física adaptada y propuestas para la construcción de unidades didácticas.
} 
- En el seguimiento de ocho meses que realizamos a un grupo de 940 maestros de educación básica, comprobamos que hubo gran interés; no obstante, encontramos debilidades en el uso de las herramientas tecnológicas; en un alto porcentaje, los maestros manifestaron temor al cambio en los sistemas de formación. Los que demostraron interés y se comunicaron con los profesores de la Universidad de Antioquia, expresaron que es necesario construir un sistema permanente de formación en el manejo de sistemas virtuales como herramienta educativa; además, los docentes no contaron con equipos computacionales propios.

- Existe un número significativo de profesores que está usando la Guía como medio de formación; sin embargo, no utilizan los medios que hemos puesto a su disposición de forma gratuita, como son el foro, el chat y el correo electrónico, aspectos que limitan las potencialidades ofrecidas por el sistema.

\section{BIBLIOGRAFÍA}

Bosco PINTO, J. (1994): «Investigación acción como práctica social», en Aportes núm. 20, Investigación Acción Participativa; aportes y desafíos, Bogotá, Dimensión educativa.

CERDA, H. (2001): Cómo elaborar proyectos. Diseño, ejecución y evaluación de proyectos sociales y educativos, Bogotá, Cooperativa Editorial Magisterio

- (2000): La creatividad en la ciencia y en la educación, Bogotá, Cooperativa Editorial Magisterio.

Contreras, O. (1998): Didáctica de la educación física. Un enfoque constructivista, INDE, Zaragoza.

GoNZÁlEZ, M. (1993): «La Educación física. Fundamentación teórica y metodológica», en Fundamentos de Educación Física para Enseñanza Primaria, vol. I, Barcelona, INDE.

INDE.

HERNÁNDEZ, J . (1994): Análisis de las estructuras del juego deportivo, Barcelona,

Hoyos, G.: «Significado de la reflexión epistemológica para la investigación acción» (documento exclusivamente para circulación interna). 


\section{D. Uribe, D. F. Gaviria}

Medellín.

FALS, O. y otros (1985): Reflexiones sobre investigación acción participativa,

FREIRE, P. (1973): Pedagogía del oprimido, México, Undécima edición, Siglo $\mathrm{XXI}$.

PEDRAZ, M. (1988): Teoría Pedagógica de la actividad física, Madrid, Gymnos.

Plan DE DeSARRollo Departamental PaRALA EdUCACIÓNFISICA (2002): «Un aporte para una cultura de la paz», Medellín, Imprenta Departamental de Antioquia.

RODRÍGUEZ, J. (1995): Deporte y ciencia, Barcelona, INDE.

RUIZ, L. (1995): Competencia motriz. Elementos para comprender el aprendizaje motor en educación física escolar, Madrid, Gymnos.

URIBE, P. I. y otros (2004): «Guía curricular. Un sistema multimedial para la formación de profesores en la educación física básica», Medellín.

VÁSQUEZ, B. (1989): La educación física en la educación básica, Madrid. Gymnos.

Página WEB: http//docencia.udea.edu.co/edufisica/guiacurricular

Correo electrónico: guiacurricular@edufisica.udea.edu.co

Foro: http://cci.udea.edu.co/foro/listaforo.php 